\title{
Support effects on the atomic structure of ultrathin silica films on metals
}

Xin Yu, Bing Yang, Jorge Anibal Boscoboinik, Shamil Shaikhutdinov,* Hans-Joachim Freund

Abteilung Chemische Physik, Fritz-Haber-Institut der Max-Planck-Gesellschaft, Faradayweg 46, 14195 Berlin

We studied the atomic structure of ultrathin silica films on $\operatorname{Pt}(111)$ in comparison with the previously studied films on $\mathrm{Mo}(112)$ and $\mathrm{Ru}(0001)$. The results obtained by scanning tunneling microscopy, photoelectron spectroscopy and infrared reflection absorption spectroscopy suggest that the metal-oxygen bond strength plays the decisive role in the atomic structure of the silica overlayers on metal substrates. Metals with high oxygen adsorption energy favor the formation of the crystalline monolayer $\mathrm{SiO}_{2.5}$ films, whereas noble metals form primarily vitreous $\mathrm{SiO}_{2}$ bilayer films. The metals with intermediate energies may form either of the structures or both coexisting. In the systems studied, the lattice mismatch plays only a minor role.

* Author to whom correspondence should be addressed. Electronic mail: shaikhutdinov@fhiberlin.mg.de 
Many modern technological applications are based on silicon dioxide $\left(\mathrm{SiO}_{2}\right)$ thin layers. In addition, thin silica films grown on metal single crystal substrates are used as model systems for studying structure-property relationships of silica and related materials using surface science techniques. ${ }^{1-5}$ One of the prominent examples in the literature relates to a crystalline silica film grown on Mo(112). ${ }^{2,6-10}$ It was shown that the ultrathin film consist of a single layer of corner sharing $\left[\mathrm{SiO}_{4}\right]$ tetrahedra forming a honeycomb-like network of a $\mathrm{SiO}_{2.5}$ stoichiometry, which is strongly bonded to Mo(112) through the Si-O-Mo linkages (the so-called "monolayer" silica film schematically shown in Fig. 1a). ${ }^{6,7}$ Recently, the preparation of $\mathrm{SiO}_{2}$ films on $\mathrm{Ru}(0001)$ has been reported, where two layers of corner sharing $\left[\mathrm{SiO}_{4}\right]$ tetrahedra form a bilayer film that is weakly bonded to $\mathrm{Ru}(0001)$ (Fig.1b). ${ }^{11,12}$ This structure resembles layered silicate minerals (diphyllosilicates) and also the inner walls of some zeolites, e.g. MCM-41. More detailed studies, using x-ray photoelectron spectroscopy (XPS), infrared reflection absorption spectroscopy (IRAS), and scanning tunneling microscopy (STM) in combination with density functional theory calculations, showed that the silica films on $\mathrm{Ru}(0001)$ first grow as a monolayer, i.e. similar to that observed on Mo(112). Only at increasing amounts of Si deposited, the film transforms into the bilayer structure. ${ }^{13}$ Depending on the preparation conditions, the bilayer film grows either in the crystalline or the vitreous state, or both coexisting. ${ }^{11,13,14}$ Regardless of the substrate (Mo or $\mathrm{Ru}$ ), thicker films grow only as vitreous silica, i.e., three-dimensional random network of $\left[\mathrm{SiO}_{4}\right]$. The principal structure of the films can be identified by IRAS, as each structure shows the individual vibrational band, i.e., $\sim 1060$ and $\sim 1135 \mathrm{~cm}^{-1}$ for monolayer films on $\mathrm{Mo}(112)$ and $\mathrm{Ru}(0001)$, respectively, reflecting the Si-O-Mo(Ru) linkages; ${ }^{6,13} \sim 1300 \mathrm{~cm}^{-1}$ for the Si-O-Si linkages between the layers in the bilayer film on $\operatorname{Ru}(0001) ;{ }^{11}$ and $\sim 1255 \mathrm{~cm}^{-1}$ for the bulk-like vitreous silica films on various metal supports as well as Si crystals. ${ }^{1,13,15-17}$

In order to understand the role of metal support in the atomic structure of the ultrathin silica films supported on, in this Letter we studied the growth of silica films on $\operatorname{Pt}(111)$, for comparison with $\mathrm{Mo}(112)$ and $\mathrm{Ru}(0001)$. The $\mathrm{Pt}(111)$ surface has been widely used as a substrate for growing transition metal oxide thin films, e.g. titania, iron oxides, ceria, etc. ${ }^{18-20}$ The surface exhibits the same symmetry, but slightly larger lattice constant than $\mathrm{Ru}(0001)$, i.e. 2.77 and $2.71 \AA$, respectively. In addition, the previously reported film preparations employed 
oxygen precovered metal surfaces prior to the Si deposition in the oxygen ambient. In this respect, Pt as the noble metal may behave differently from the Mo and Ru supports.

The experiments were performed in an UHV chamber (base pressure $5 \times 10^{-10} \mathrm{mbar}$ ) equipped with LEED, XPS, IRAS and STM. The Pt(111) crystal was mounted on the Omicron sample holder. The temperature was measured by a Type K thermocouple spot-welded to the edge of the crystal. The clean $\mathrm{Pt}(111)$ surface was obtained by cycles of $\mathrm{Ar}^{+}$sputtering and annealing in UHV to $1273 \mathrm{~K}$. The preparation of silica films on $\mathrm{Pt}(111)$ nearly copies that of on $\mathrm{Ru}(0001) .{ }^{11-14}$ First, we prepared the $\mathrm{O}-(2 \times 2)-\mathrm{Pt}(111)$ surface by exposing to $3 \times 10^{-6} \mathrm{mbar}_{2}$ at 1200 K for 5 min and cooling to the room temperature in the same ambient. Then Si (99.99\%) was deposited using e-beam assisted evaporator (EMT3, Omicron) in $1.5 \times 10^{-6} \mathrm{mbar}_{2}$ onto the substrate kept at $\sim 100 \mathrm{~K}$. Final oxidation was performed in $1 \times 10^{-5} \mathrm{mbar}_{2}$ at $\sim 1200 \mathrm{~K}$. The amount of $\mathrm{Si}$ at the surface was measured by XPS using the well-established $\mathrm{SiO}_{2.5} / \mathrm{Mo}(112)$ structure $^{8}$ as a reference. For simplicity, the Si coverage is presented in the text in monolayer equivalents (MLE) such that 2 MLE corresponds to the amount of Si in the ideal bilayer film. The XPS spectra were referenced by setting the $\mathrm{Au} 4 \mathrm{f}_{7 / 2}$ level to $84.0 \mathrm{eV}$ measured on a clean gold foil.

First we address the structure of the 2 MLE silica films on $\operatorname{Pt}(111)$. The XPS measurements revealed only one chemical state of silicon, with a binding energy (BE) of the Si2p core level $102.8 \mathrm{eV}$ that falls in the range of $\mathrm{Si}^{4+}$. For comparison, the value $102.5 \mathrm{eV}$ was obtained on $\mathrm{SiO}_{2} / \mathrm{Ru}(0001) .{ }^{11,12}$ The O1s region showed a main peak at $531.9 \mathrm{eV}$ (531.7 eV for $\mathrm{SiO}_{2} / \mathrm{Ru}(0001)$ ) with a small shoulder at $530.1 \mathrm{eV}$ contributing only $\sim 6 \%$ to the overall signal intensity. The XPS data are very similar to those obtained for the bilayer film on $\mathrm{Ru}(0001)$, except that the $\sim 530 \mathrm{eV}$ shoulder is less prominent on Pt(111). The difference can, in principle, be assigned to the lower affinity of $\mathrm{Pt}(111)$ towards oxygen since this signal is associated with the oxygen atoms directly adsorbed on the metal surface underneath a film ${ }^{12}$ and/or in holes exposing a metal support.

The presence of small holes in this sample is seen in the large-scale STM image (Fig. 2a), which otherwise shows an uniformly covered film with relatively wide terraces separated by the monoatomic steps of $\operatorname{Pt}(111)$. The holes are $\sim 2 \AA$ in depth, which is considerably lower than $\sim 5$ 
$\AA$ observed for the bilayer films on $\mathrm{Ru}(0001) \cdot{ }^{13,14}$ On the other hand, the atomically resolved STM image shown in Fig. $2 \mathrm{~b}$ is virtually identical to those obtained for the vitreous silica bilayer film on $\mathrm{Ru}(0001)$, where a random two-dimensional network of corner-sharing $\left[\mathrm{SiO}_{4}\right]$ tetrahedra results in a variety of $\mathrm{N}$-membered rings, with $\mathrm{N}$ varied between 4 and $9 .{ }^{14}$ Also, LEED inspection showed only $\mathrm{Pt}(111)$ diffraction spots together with a diffuse $(2 \times 2)$ ring, thus indicating the lack of long-range ordering. In order to identify the principal structure of the film one has to invoke IRAS since, as mentioned in the introduction, each silica structure exhibits individual vibrational bands.

The IRA-spectrum of this film, exhibiting only two sharp bands at 1294 and $690 \mathrm{~cm}^{-1}$ (Fig. 3), is virtually identical to the one observed on the bilayer films on $\mathrm{Ru}(0001)$ (1302 and 692 $\left.\mathrm{cm}^{-1}\right) .{ }^{11}$ This finding straightforwardly leads to the conclusion that the film is bilayered in nature, and it grows in the vitreous state as judged by LEED and STM. Note, that on $\mathrm{Ru}(0001)$ it was possible to grow crystalline films as well, e.g. using a low cooling rate after high temperature annealing. ${ }^{11,13,14}$ In the case of $\operatorname{Pt}(111)$, however, the crystalline films have not yet been observed at any combination of preparation parameters studied. This issue needs further studies and is beyond the scope of this paper.

Now we address the results for the 1 MLE films, which were prepared by reducing the amounts of Si deposited, while keeping all other parameters the same. The IRAS study immediately showed the bilayer structure of the resulted film (Fig. 3). It is clear that the intensity of the IRA bands at 1294 and $690 \mathrm{~cm}^{-1}$ simply scales with the Si coverage and does not show any feature at $1000-1100 \mathrm{~cm}^{-1}$ otherwise expected for the monolayer film. The scaling behavior is also observed in XP-spectra, although the $\mathrm{O} / \mathrm{Pt}(111)$ signal at $530 \mathrm{eV}$ becomes more pronounced since the sample must expose larger fraction of the bare Pt surface.

Indeed, an STM image (Fig. 4) of the 1 MLE film shows that only half of the entire surface is covered by silica. The surface exposes two-dimensional islands, showing basically the same vitreous structure (zoomed in the inset in Fig. 4a) as in the case of the 2 MLE films (cf Fig. 2b). The apparent height of the islands, i.e. in the order of $2 \AA$ (Fig. 4b), is much lower than the geometrical film thickness, $\sim 5 \AA$, but definitely higher than $\sim 1.4 \AA$ observed for the monolayer films on $\mathrm{Ru}(0001) .{ }^{13}$ Tentatively, we assigned this behavior to the electronic effects frequently 
observed for STM imaging of metal-oxide systems, which, in addition, may be bias- and polarity-dependent. Interestingly, the islands are bridged by narrow stripes of the same height running primarily along the principal crystallographic directions of $\operatorname{Pt}(111)$. Most of these “bridges” are $\sim 7 \AA$ in width, although $\sim 4 \AA$ and $\sim 10 \AA$ wide lines were observed as well. To some extent, these stripes resemble one-dimensional silica rows formed at low Si coverage on $\operatorname{Mo}(112),{ }^{21}$ although the atomic structure could not be identified here. Nonetheless, the above presented XPS, STM and IRAS results show that ultrathin silica films on Pt(111) grows exclusively in the bilayer form.

Now we are in position to compare the principal structures of the ultrathin silica films grown on $\mathrm{Mo}(112), \mathrm{Ru}(0001)$ and $\mathrm{Pt}(111)$. On all supports, the films are formed by a network of corner sharing $\left[\mathrm{SiO}_{4}\right]$ tetrahedra. On $\mathrm{Mo}(112)$, the silica grows only as a monolayer strongly bonded to Mo via the Si-O-Mo linkages. On Ru(0001), both the monolayer, bonded through the Si-O-Ru linkage, and the bilayer, weakly bonded to $\mathrm{Ru}$, structures are observed, depending on the Si coverage. Irrespective of the Si coverage, only bilayer films grow on $\mathrm{Pt}(111)$. Obviously, this trend correlates with the oxygen affinity to the metal support. Indeed, heats of dissociative adsorption of oxygen are of $-544,-220$ and $-133 \mathrm{~kJ} / \mathrm{mole}$ for the $\mathrm{Mo}, \mathrm{Ru}$, and $\mathrm{Pt}$, respectively, ${ }^{22}$ which correlate with standard heats of formation for $\mathrm{MoO}_{2}, \mathrm{RuO}_{2}$, and $\mathrm{PtO}_{2}(-588,-153$, and -71 $\mathrm{kJ} /$ mole, respectively). ${ }^{23}$ Therefore, the SiO-Mo bond is much stronger than the SiO-Pt bond and as such will favor the monolayer structure, whereas on Pt silica forms "closed shell”, bilayer structure terminated by the fully saturated oxygen layer on either side. Ruthenium exhibits the intermediate properties and forms both mono- and bi-layer structures. Very recently, it has been reported the (accidental) formation of silica bilayer on graphene ${ }^{24}$ that represents an oxygenresistant, weakly-bonded support. This finding is in full agreement with the trend observed above on metals.

One may argue that the lattice mismatch between an oxide film and a metal substrate, which is obviously different for the metals studied, may also play a role. The lattice constant of the unsupported, free-standing silica bilayer is computed to be $5.32 \AA$. $^{9}$ When supported on $\mathrm{Ru}(0001)$ (lattice constant $2.71 \AA$ ) and $\operatorname{Pt}(111)$ (lattice constant $2.77 \AA$ ) the most natural structure for the silica film to accommodate these metal supports is the $(2 \times 2)$ structure resulting 
in a periodicity of $5.42 \AA$ and $5.54 \AA$, respectively, that is indeed observed by LEED and STM. From this point of view, $\mathrm{Ru}(0001)$ is better suited than $\mathrm{Pt}(111)$ as the lattice mismatch is smaller. This may explain why on $\mathrm{Ru}(0001)$, and not on $\mathrm{Pt}(111)$, crystalline films may be grown under certain conditions. ${ }^{11,13}$ On the other hand, Mo(112) shares a rectangular unit cell (5.46 $\AA$ × 8.92 Å) which hardly fit a hexagonal silica overlayer. As a result, the silica films on $\mathrm{Mo}(112)$ forms the $\mathrm{c}(2 \times 2)$ structure which is accompanied by silica lattice extension along the Mo[-1-11] direction to $5.46 \AA$ and shortening along the Mo[-311] direction to $5.2 \AA$. However, despite this distortion, the monolayer silica film on $\mathrm{Mo}(112)$ is perfectly ordered on the large scale, ${ }^{6,8}$ whereas on $\mathrm{Ru}(0001)$ the monolayer film forms multi-domain structure with a high density of domain boundaries. ${ }^{13}$ Therefore, it is the strong SiO-Mo bond that stabilizes the well-ordered monolayer structure despite of the energy cost for the silica lattice distortion.

In summary, we studied the preparation and the atomic structure of ultrathin silica films on $\mathrm{Pt}(111)$ in comparison with the previously studied $\mathrm{Mo}(112)$ and $\mathrm{Ru}(0001)$. The results point to that the metal-oxygen bond strength plays the decisive role in the atomic structure of the silica overlayers on metal substrates. Metals with the high oxygen adsorption energy favor the formation of the crystalline monolayer $\mathrm{SiO}_{2.5}$ films, whereas noble metals form primarily vitreous $\mathrm{SiO}_{2}$ bilayer films. The metals with intermediate energies may form either of the structures or both coexisting. In the systems studied, the lattice mismatch plays only a minor role.

\section{References}

${ }^{1}$ X. Xu, and D.W. Goodman, Appl. Phys. Lett. 61, 774 (1992).

${ }^{2}$ T. Schroeder, M. Adelt, B. Richter, M. Naschitzki, M. Bäumer, and H.-J. Freund, Surf. Rev. Lett. 7, 7 (2000). DOI No: 10.1142/S0218625X00000038

${ }^{3}$ M.S. Chen, A.K. Santra, and D.W. Goodman, Phys. Rev. B 69, 155404 (2004).

${ }^{4}$ M. Kundu, and Y. Murata, Appl. Phys. Lett. 80, 1921 (2002).

${ }^{5}$ Z. Zhang, Z.Jiang, Y. Yao, D. Tan, Q. Fu, and X. Bao, Thin Solid Films 516, 3741 (2008).

${ }^{6}$ J. Weissenrieder, S. Kaya, J.-L. Lu, H.-J. Gao, S. Shaikhutdinov, H.-J. Freund, M.M. Sierka, T.K. Todorova, and J. Sauer, Phys. Rev. Lett. 95, 076103 (2005).

${ }^{7}$ L. Giordano, D. Ricci, G. Pacchioni, and P. Ugliendo, Surf. Sci. 584, 225 (2005). 
${ }^{8}$ S. Kaya, M. Baron, D. Stacchiola, J. Weissenrieder, S. Shaikhutdinov, T. K. Todorova, M. Sierka, J. Sauer, and H.-J. Freund, Surf. Sci. 601, 4849 (2007).

${ }^{9}$ U. Martinez, L. Giordano, and G. Pacchioni, J. Phys. Chem. B 110, 17015 (2006).

10 J. Seifert, D. Blauth, and H. Winter, Phys. Rev. Lett. 103, 017601 (2009).

${ }^{11}$ D. Löffler, J. J. Uhlrich, M. Baron, B. Yang, X. Yu, L. Lichtenstein, L. Heinke, C. Büchner, M. Heyde, S. Shaikhutdinov, H.-J. Freund, R. Włodarczyk, M. Sierka, and J. Sauer, Phys. Rev. Lett. 105, 146104 (2010).

${ }^{12}$ R. Włodarczyk, M. Sierka, J. Sauer, D. Löffler, J. J. Uhlrich, X. Yu, B. Yang, I. M. N. Groot, S. Shaikhutdinov, and H.-J. Freund, Phys. Rev. B 85, 085403 (2012).

13 B. Yang, X. Yu, J.A. Boscoboinik, L. Lichtenstein, M. Heyde, B. Kaden, R. Włodarczyk, M. Sierka, J. Sauer, S. Shaikhutdinov, and H.-J. Freund, unpublished.

Thin silica films on $\mathrm{Ru}(0001)$ : Monolayer, bilayer and three-dimensional networks of $\left[\mathrm{SiO}_{4}\right]$ tetrahedra.

${ }^{14}$ L. Lichtenstein, C. Büchner, B. Yang, S. Shaikhutdinov, M. Heyde, M. Sierka, R. Włodarczyk, J. Sauer, and H.-J. Freund, Angew. Chem. Int. Ed. 51, 404 (2012).

15 J.E. Olsen, and F. Shimura, Appl. Phys. Lett. 53, 1934 (1988).

${ }^{16}$ K.T. Queeney, M.K. Weldon, J.P. Chang, Y.J. Chabal, A.B. Gurevich, J. Sapjeta, and R.L. Opila, J. Appl. Phys. 87, 1322 (2000).

17 D.J. Stacchiola, M. Baron, S. Kaya, J. Weissenrieder, S. Shaikhutdinov, and H.-J. Freund, Appl. Phys. Lett. 92, 011911 (2008).

${ }^{18}$ P. Luches, F. Pagliuca, and S. Valeri, J. Phys. Chem. C 115, 10718 (2011).

${ }^{19}$ W. Weiss, and M. Ritter, Phys. Rev. B 59, 5201 (1999).

${ }^{20}$ U. Berner, and K.-D. Schierbaum, Phys. Rev. B 65, 235404 (2002).

${ }^{21}$ J. L. Lu, S. Kaya, J. Weissenrieder, T. K. Todorova, M. Sierka, J. Sauer, H. J. Gao, S. Shaikhutdinov, and H. -J. Freund, Surf. Sci. Lett. 600, 164 (2006).

${ }^{22}$ C.N.R. Rao, P. Vishnu Kamath, and S. Yashonath, Chem. Phys. Lett. 88, 13 (1982).

${ }^{23}$ B. A. Staskiewicz, J. R. Tucker, and P. E. Snyder, J. Am. Chem. Soc. 77, 2987 (1955).

${ }^{24}$ P.Y. Huang, S. Kurasch, A. Srivastava, V. Skakalova, J. Kotakoski, A. V. Krasheninnikov, R. Hovden, Q. Mao, J. C. Meyer, J. Smet, D. A. Muller, and U. Kaiser, Nano Letters 12, 1081 (2012). 


\section{Figure captions}

Figure 1. Schematic representation of a monolayer (a) and a bilayer (b) film on a metal support. Top and cross views are shown. Position of the $\mathrm{Si}$ ions in $\left[\mathrm{SiO}_{4}\right]$ tetrahedra is indicated by dots.

Figure 2. Large-scale (a) and atomically resolved (b) STM images of the 2 MLE silica films on Pt(111). Tunneling parameters: bias $4.4 \mathrm{~V}$ and current $0.1 \mathrm{nA}$ (a); $1.3 \mathrm{~V}, 0.13 \mathrm{nA}$ (b).

Figure 3. IRA-spectra of 1 MLE (top) and 2 MLE (bottom) films grown on $\operatorname{Pt}(111)$.

Figure 4. (a) STM image of the 1 MLE film on Pt(111). Height profile along the A-B line, indicated in (a), is shown in (b). The inset shows a close-up image demonstrating the vitreous state of silica islands (cf Fig. 2b). The arrow indicates silica stripes bridging the islands. (Tunneling parameters $0.8 \mathrm{~V}, 0.06 \mathrm{nA}$ ). 